\title{
Efficacy and Safety of Silodosin and Dutasteride Combination Therapy in Acute Urinary Retention due to Benign Prostatic Hyperplasia: A Single-Arm Prospective Study
}

\author{
Kazuhisa Hagiwara, Takuya Koie, Hiromichi Iwamura, Atsushi Imai, Shingo Hatakeyama, \\ Takahiro Yoneyama, Yasuhiro Hashimoto, and Chikara Ohyama
}

Department of Urology, Hirosaki University Graduate School of Medicine, Hirosaki 036-8562, Japan

Correspondence should be addressed to Yasuhiro Hashimoto; bikkuri@opal.plala.or.jp

Received 18 January 2016; Accepted 24 March 2016

Academic Editor: Masaki Shiota

Copyright (C) 2016 Kazuhisa Hagiwara et al. This is an open access article distributed under the Creative Commons Attribution License, which permits unrestricted use, distribution, and reproduction in any medium, provided the original work is properly cited.

\begin{abstract}
This study aimed to assess the efficacy of combination therapy with dutasteride and silodosin in patients with acute urinary retention (AUR) caused by benign prostatic hyperplasia (BPH). Eighty consecutive patients with a first episode of AUR were enrolled in this study. All patients received silodosin $8 \mathrm{mg}$ and dutasteride $0.5 \mathrm{mg}$ daily. Trial without catheter (TWOC) was attempted every 2 weeks until 12 weeks after the initiation of medication. The primary endpoint was the rate of catheter-free status at 12 weeks. Voided volume (VV), postvoid residual urine (PVR), uroflowmetry, International Prostatic Symptoms Score (IPSS), and quality of life due to urinary symptoms (IPSS-QOL) were also measured. All patients were followed up for more than 12 weeks and were included in this analysis. The success rate of TWOC at 12 weeks was $88.8 \%$. VV and maximum urinary flow rate were significantly higher at 2 , 4,8 , and 12 weeks compared with the time of AUR $(P<0.001)$. IPSS and IPSS-QOL were significantly lower at $2,4,8$, and 12 weeks compared with the time of AUR $(P<0.001)$. In conclusion, a combination of dutasteride and silodosin therapy may be effective and safe for patients with AUR due to BPH.
\end{abstract}

\section{Introduction}

Acute urinary retention (AUR) is a common urological emergency and is defined as a sudden and painful inability to pass urine [1]. In most male patients, AUR is attributed to the natural history of benign prostatic hyperplasia (BPH) [2]. Baseline variables for AUR patients with BPH are old age, severe lower urinary tract symptoms (LUTS), low peak flow rate, increased postvoid residual urine (PVR), enlarged prostate, and high serum prostate-specific antigen (PSA) levels $[3,4]$.

Immediate management of AUR requires complete bladder decompression by inserting an indwelling urinary catheter. Although a trial without catheter (TWOC) is the standard of care for AUR [5], TWOC typically involves removing the catheter after 3 days, which allows only
$23 \%-40 \%$ of patients to void successfully $[5,6]$. TWOC in which catheter removal followed 2-3 days of $\alpha$-blocker (AB) improves success rates [7]. Kumar et al. reported that silodosin significantly increased the chances of successful TWOC after AUR [8]. Alternatively, a $5 \alpha$-reductase inhibitor (5-ARI) alone or combined with $\mathrm{AB}$ is a treatment option in patients with LUTS due to BPH [9]. Long-term combination therapy with $A B$ and 5-ARI has been shown to reduce progression to AUR and the need for surgery, in addition to providing symptomatic relief $[10,11]$. However, the utility of combination therapy with silodosin and dutasteride in TWOC after AUR has not been studied to date. The aim of this prospective single-arm study was to assess the impact of $8 \mathrm{mg}$ silodosin twice daily and dutasteride $0.5 \mathrm{mg}$ once daily on the outcome of TWOC after a first episode of AUR. 


\section{Materials and Methods}

2.1. Patient Selection. The study protocol and informed consent documents were reviewed and approved by the Hirosaki University Institutional Review Board. All patients gave their written informed consent to participate in the trial.

All consecutive patients presenting with their first episode of spontaneous AUR were enrolled. Exclusion criteria were urinary tract infections, urological tumors, clot retention, urethral stricture, chronic urinary retention, urolithiasis, drug abuse, neurogenic lower urinary tract dysfunction, and a history of prostatic surgery or urological treatments.

Clinical details including medical history, date of catheterization, retention urine volume, digital rectal examination (DRE) findings, and prostate volume were recorded at the time of AUR. Voided volume (VV), PVR, uroflowmetry, LUTS (graded according to International Prostatic Symptoms Score (IPSS)), and quality of life due to urinary symptoms (IPSS-QOL) were also measured every 2 weeks. Serum PSA levels were measured at the time of AUR before catheterization and 12 weeks after the initiation of medication.

2.2. Treatment. After initial management of AUR with immediate bladder decompression by inserting an indwelling urinary catheter, all patients were given $4 \mathrm{mg}$ oral silodosin tablet twice daily and a $0.5 \mathrm{mg}$ dutasteride capsule once daily until catheter removal.

A TWOC was attempted every 2 weeks until 12 weeks after the initiation of medication. In this study, the catheter was removed after the instillation of $200 \mathrm{~mL}$ normal saline. The patients were considered to have achieved a catheterfree status (CFS) if they could void voluntarily with a PVR of $<100 \mathrm{~mL}$ and did not require recatheterization in the next $24 \mathrm{~h}$.

2.3. Endpoints and Statistical Analysis. The primary endpoint was the proportion of successful TWOCs. The secondary endpoints were changes over time in IPSS score and PVR. Other outcomes measured were the cumulative incidence of invasive treatments related to $\mathrm{BPH}$, including transurethral resection of the prostate and stenting and changes over time in the serum PSA levels and prostate volume.

Data were analyzed using SPSS 22 statistical software (IBM Corp., Armonk, NY, USA). Statistical comparisons were made using the chi-square test for qualitative variables and Student's $t$-test for quantitative variables. The influence of study variables on the TWOC success rate was tested using logistic regression methods. All $P$ values were 2 -sided, and the significance level was set at $<0.05$.

\section{Results}

In total, 80 patients presenting with a painful AUR between September 2010 and June 2013 from public (50\%) or private (50\%) healthcare practices were enrolled. All the patients completed the trial protocol. The clinical characteristics of the enrolled patients are listed in Table 1 . All patients completed the trial protocol and experienced no adverse event.
TABLE 1: Clinical characteristics of the study participants.

\begin{tabular}{lc}
\hline Age (years) & $75.0(68.8-81.3)$ \\
$\quad$ Median (IQR) & \\
Retention volume (mL) & $500(375-1000)$ \\
$\quad$ Median (IQR) & $26(16-31)$ \\
IPSS & \\
$\quad$ Median (IQR) & $5(5-6)$ \\
IPSS-QOL & \\
$\quad$ Median (IQR) & $5.80(3.15-12.70)$ \\
PSA (ng/mL) & \\
$\quad$ Median (IQR) & \\
Prostate volume (mL) & $46.4(34.9-69.6)$ \\
$\quad$ Median (IQR)
\end{tabular}

IQR, interquartile range; IPSS, International Prostatic Symptoms Score; IPSS-QOL, quality of life due to urinary symptoms; PSA, prostate-specific antigen.

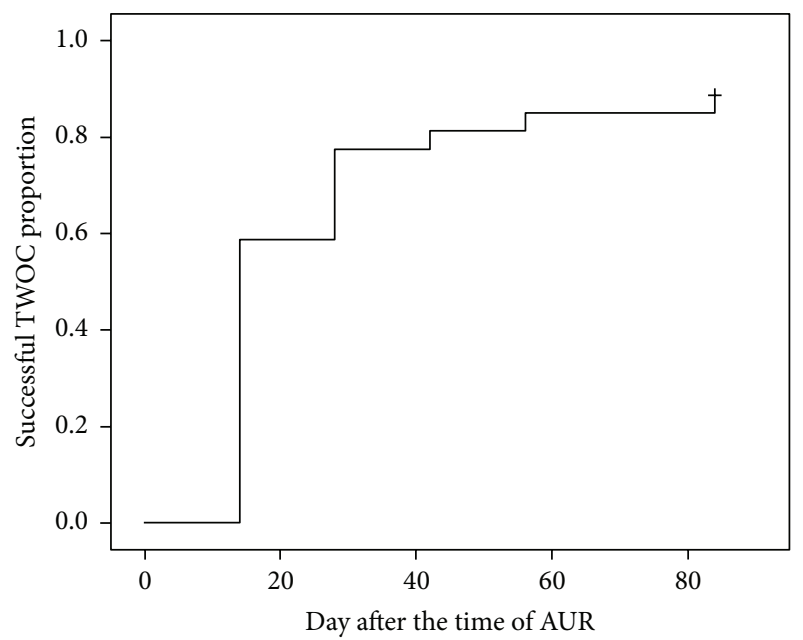

FIGURE 1: Kaplan-Meier estimate of successful trial without catheter for patients with acute urinary retention. The success rate of trial without catheter was $88.8 \%$ at 12 weeks.

The success rate of TWOC was $88.8 \%$ at 12 weeks (Figure 1). Nine patients (11.2\%) had an indwelling catheter owing to voiding failure. Of 71 patients with successful TWOC, 7 (9.9\%) had a second episode of AUR within the first 3 months after the successful TWOC. Eight (10\%) patients, including 4 with a successful TWOC, required BPH surgery.

Regarding VV, PVR, and uroflowmetry, 35 patients were evaluated at 2 weeks, 33 patients at 4 weeks, 10 patients at 8 weeks, and 36 patients at 12 weeks. The chronological changes in VV and PVR are shown in Figure 2. VV gradually increased throughout the entire evaluation period and was significantly higher at 2, 4, 8, and 12 weeks compared with the time of AUR ( ${ }^{*} P<0.001$; Figure $\left.2(\mathrm{a})\right)$. The median PVR at 2 weeks was $63 \mathrm{~mL}$ (interquartile rate (IQR) 14-63), which was maintained throughout the entire evaluation period $\left({ }^{*} \mathrm{P}<\right.$ 0.001; Figure 2(b)). The maximum urinary flow rate (Qmax) also gradually increased throughout (Figure 3). Qmax was 


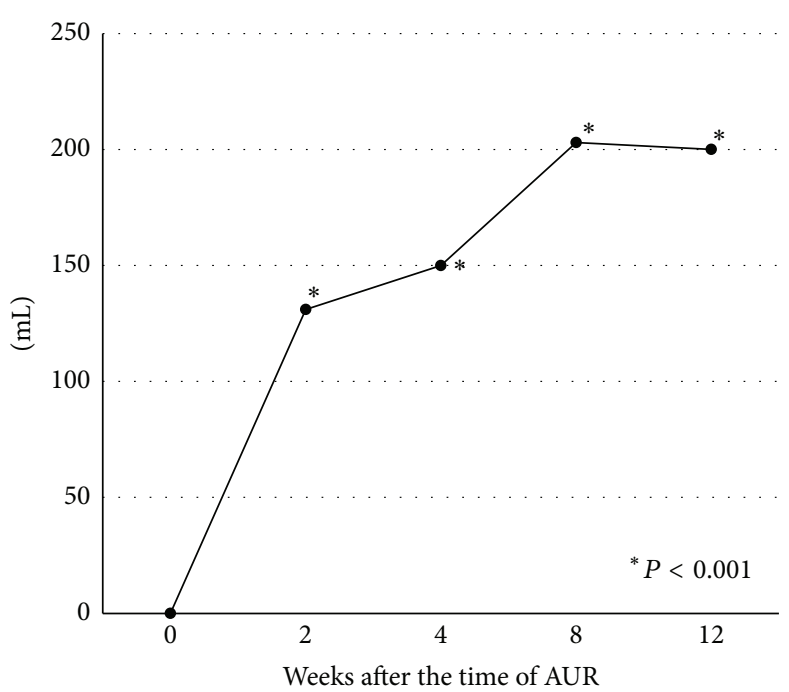

(a) Voided volume

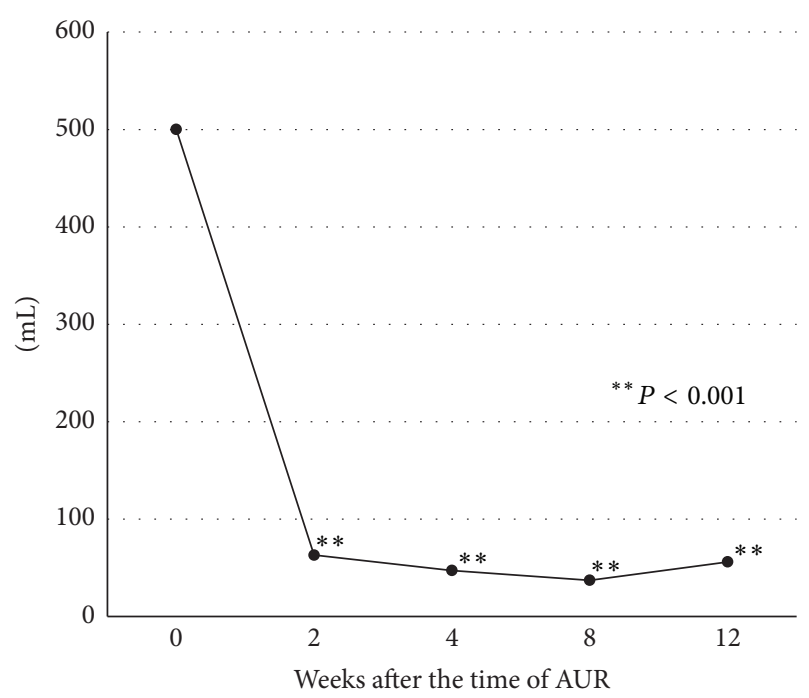

(b) Postvoid residual urine

FIGURE 2: The chronological changes in voided volume (a) and postvoid residual urine (b). (a) Voided volume gradually increased throughout the entire evaluation period and was significantly higher at 2, 4,8, and 12 weeks than that at the time of acute urinary retention $\left({ }^{*} P<0.001\right)$. (b) Postvoid residual urine at 2 weeks was $63 \mathrm{~mL}$ and significantly lower at 2, 4, 8, and 12 weeks than that at the time of acute urinary retention $\left({ }^{* *} P<0.001\right)$.

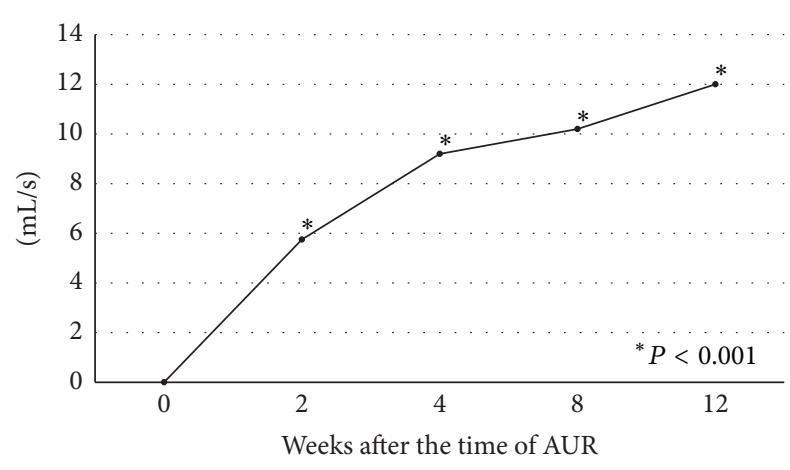

FIGURE 3: Chronological changes in maximum urinary flow rate. Maximum urinary flow rate was significantly higher at 2, 4, 8, and 12 weeks than that at the time of acute urinary retention $\left({ }^{*} P<0.001\right)$.

significantly higher at 2, 4, 8, and 12 weeks compared with the time of AUR $\left({ }^{*} P<0.001\right)$.

The chronological changes in IPSS and IPSS-QOL are shown in Figure 4. IPSS at 2 weeks was 8 (IQR 7-9), which was maintained throughout the entire evaluation period $\left({ }^{*} P<0.001\right.$; Figure $\left.4(\mathrm{a})\right)$. IPSS-QOL gradually decreased throughout the entire evaluation period and was significantly lower at 2, 4, 8, and 12 weeks compared with the time of AUR $\left({ }^{* *} P<0.001\right.$; Figure $\left.4(\mathrm{~b})\right)$.

The median serum PSA level was $5.8 \mathrm{ng} / \mathrm{mL}$ at the time of AUR and $3.3 \mathrm{ng} / \mathrm{mL}$ at 12 weeks. Median prostate volume was $46.4 \mathrm{~mL}$ at the time of AUR and $38.1 \mathrm{~mL}$ at 12 weeks. Serum PSA level $(P=0.003)$ and prostate volume $(P<0.001)$ were significantly decreased at 12 weeks compared with the time of AUR.

\section{Discussion}

AUR is a common urological emergency in men. The immediate treatment of AUR is catheterization followed by TWOC after a variable interval. The management of AUR is not standardized because of a lack of existing guidelines, and important differences exist among institutions and countries with regard to the duration of catheterization and management of TWOC. Until recently, standard management in patients with AUR was prostatic surgery within a few days or weeks after a first AUR episode. However, patients who underwent AUR-related emergency prostatectomy with a urinary catheter were at a greater risk of peri- and postoperative complications, including sepsis or death, compared with those who underwent elective prostatectomy for symptoms alone [12]. The relative risk was 1.8 for perioperative complications, 1.6 for postoperative complications, and 3.3 overall for hospital death, with a relative risk of 26.6 at 30 days and 4.4 at 90 days [12]. In addition, Murray et al. reported that up to 23\% patients with AUR did not require prostatectomy based on urodynamic assessment [13]. In contrast, TWOC involves removing the catheter after $1-3$ days, which allows $23 \%-40 \%$ of patients to void successfully $[5,6]$.

$\mathrm{ABs}$ are recommended as first-line treatment for LUTS with moderate to severe symptoms due to $\mathrm{BPH}$ [14]. Currently, TWOC after $A B$ therapy is also the recommended treatment option for patients with AUR. The large randomized, double-blind, placebo-controlled alfuzosin in another AUR study investigated the impact of $A B$ on the outcome of TWOC [7]. Three hundred sixty patients with a first episode of AUR-related BPH were randomized to receive $10 \mathrm{mg}$ alfuzosin once daily or placebo for 2-3 days following 


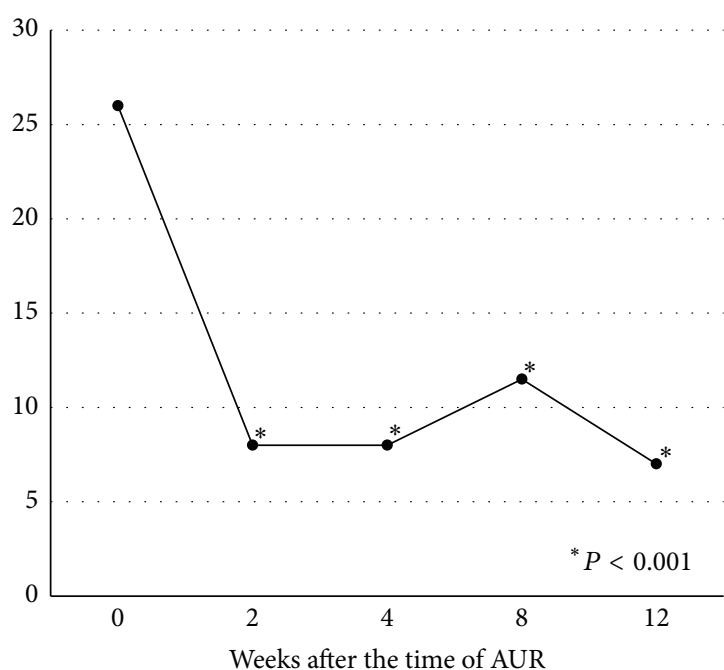

(a) IPSS

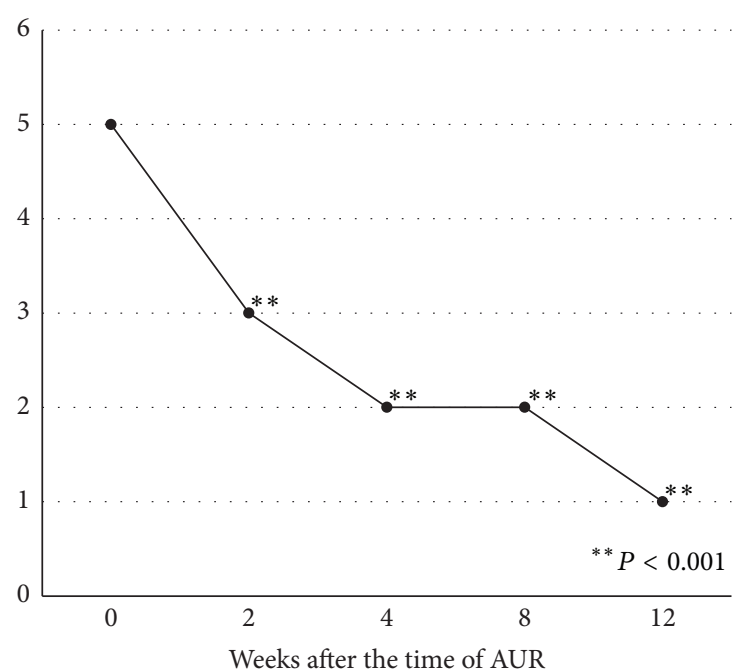

(b) IPSS-QOL

FIgURE 4: Chronological changes in International Prostate Symptom Score (a) and quality of life due to urinary symptoms (b). (a) International Prostate Symptom Score was significantly improved at 2, 4, 8, and 12 weeks compared with that at the time of acute urinary retention $\left({ }^{*} P<0.001\right)$. (b) Quality of life due to urinary symptoms was significantly improved at $2,4,8$, and 12 weeks compared with that at the time of acute urinary retention $\left({ }^{* *} P<0.001\right)$.

catheterization [7]. The successful voiding rate was significantly higher in patients treated with alfuzosin than in those treated with placebo (62\% versus $48 \%$, resp.; $P=$ 0.002) [7]. Various clinical trials have demonstrated that ABs, including tamsulosin and alfuzosin, are effective and safe in AUR management, with successful removal percentages that range from $48 \%$ to $70 \%$ [15]. Kumar et al. reported that patients receiving silodosin were twice as likely to void successfully as those receiving placebo [8]. The success rate of TWOC in the silodosin group was slightly better than those reported after tamsulosin or alfuzosin in TWOC [6, $7,15]$. One reason for this may be that silodosin's $\alpha-1 \mathrm{~A}: \alpha-1 \mathrm{~B}$ binding ratio is extremely high $(162: 1)$, leading to its selective action in the lower urinary tract with minimal side effects on blood pressure regulation [8]. Therefore, silodosin has a good uroselectivity compared with other $\mathrm{ABs}$ and may have better efficacy than tamsulosin $[16,17]$.

Several reports suggested that the post-AUR use of $A B$ prevents recurrence and increases the success rate of selfvoiding $[7,18]$. However, despite continuing medication, $17.1 \%$ of patients required surgical treatment during 6 months [18]. AUR patients with large prostate volume were most at risk for recurrent AUR or prostatic surgery [3]. Recently, combination therapy with 5-ARI and $\mathrm{AB}$ provided significantly greater benefit than either monotherapy for various outcomes in patients with LUTS due to BPH and prostatic enlargement [10, 11]. The Medical Therapy of Prostatic Symptoms study showed (with a mean follow-up period of 4.5 years) that combination of finasteride and doxazosin therapy significantly reduced the risk of overall clinical progression of BPH, AUR, and need for invasive therapy in patients with LUTS due to BPH [10]. McConnell et al. also reported that the reduction in the risk of AUR and the need for invasive therapy throughout the study may be attributed to a reduction in prostate size [10]. According to the combination of Avodart ${ }^{\circledR}$ and tamsulosin study, dutasteride and tamsulosin combination therapy significantly reduced the relative risk of AUR- or BPH-related surgery over 4 years by $66 \%$ compared with tamsulosin monotherapy [11]. In addition, combination therapy significantly decreased the relative risk of clinical progression and symptom deterioration in patients with IPSS $\geq 4$ points [11]. These data suggest that combination of dutasteride and tamsulosin therapy in patients with LUTS due to $\mathrm{BPH}$ provided rapid and durable symptom benefit and reduced the long-term risk of BPH clinical progression [11].

Our findings were limited because this was a nonrandomized single-arm study with a relatively small sample size. In this study, the success rate of TWOC was $88.8 \%$ at 12 weeks and achieved relatively higher CFS than previous reports $[7,8,15]$; furthermore, relatively few patients with a successful TWOC required surgical treatment compared with those in previous reports [7]. In addition, Qmax, IPSS, and IPSSQOL were significantly improved and remained favorable throughout the 12 -week study.

These results suggest that combination therapy with dutasteride and silodosin could have potential to achieve a relatively high success rate of TWOC. A prospective randomized trial is necessary to determine whether the combination therapy is superior to single-agent therapy.

\section{Competing Interests}

The authors declare that they have no competing interests.

\section{Acknowledgments}

The authors would like to acknowledge the following coordinating investigators: Shin Itoh (Ai Clinic), Takeshi 
Yanagisawa (Aomori Rosai Hospital), Masahiko Kitagawa (Kitagawa Urology Clinic), Tatsuya Kudoh (Kudoh Clinic), Yutaka Tachiki (Tachiki Clinic), Shigeya Togashi (Japan Community Healthcare Organization Akita Hospital), Keiya Miki (Miki Clinic), Kazuaki Yoshikawa (Mutsu Hospital), Noritaka Kamimura (Hirosaki National Hospital), Toshiaki Kawaguchi (Aomori Prefectural Central Hospital), Yuji Yagihashi (Yagihashi Kidney-Urology Clinic), Shin-ya Takahashi (Aomori City Hospital), Atsushi Kyan (Shirakawa General Hospital), Yasuo Kusumi (Kusumi Urology Clinic), and Atsushi Tsukui (Aomori Jikeikai Hospital).

\section{References}

[1] M. Emberton and K. Anson, "Fortnightly review: acute urinary retention in men: an age old problem," British Medical Journal, vol. 318, no. 7188, pp. 921-925, 1999.

[2] J. M. Fitzpatrick, F. Desgrandchamps, K. Adjali et al., "Management of acute urinary retention: a worldwide survey of 6074 men with benign prostatic hyperplasia," BJU International, vol. 109, no. 1, pp. 88-95, 2012.

[3] S. J. Jacobsen, D. J. Jacobson, C. J. Girman et al., "Natural history of prostatism: risk factors for acute urinary retention," Journal of Urology, vol. 158, no. 2, pp. 481-487, 1997.

[4] C. Kolman, C. J. Girman, S. J. Jacobsen, and M. M. Lieber, "Distribution of post-void residual urine volume in randomly selected men," Journal of Urology, vol. 161, no. 1, pp. 122-127, 1999.

[5] R. Manikandan, S. J. Srirangam, P. H. O'Reilly, and G. N. Collins, "Management of acute urinary retention secondary to benign prostatic hyperplasia in the UK: a national survey," $B J U$ International, vol. 93, no. 1, pp. 84-88, 2004.

[6] S. A. McNeill, P. D. Daruwala, I. D. C. Mitchell, M. G. Shearer, and T. B. Hargreave, "Sustained-release alfuzosin and trial without catheter after acute urinary retention; a prospective, placebo-controlled trial," BJU International, vol. 84, no. 6, pp. 622-627, 1999.

[7] S. A. McNeill and T. B. Hargreave, "Alfuzosin once daily facilitates return to voiding in patients in acute urinary retention," Journal of Urology, vol. 171, no. 6, part 1, pp. 2316-2320, 2004.

[8] S. Kumar, D. P. Tiwari, R. Ganesamoni, and S. K. Singh, "Prospective randomized placebo-controlled study to assess the safety and efficacy of silodosin in the management of acute urinary retention," Urology, vol. 82, no. 1, pp. 171-175, 2013.

[9] R. Morlock, B. Goodwin, G. Gomez Rey, and M. Eaddy, "Clinical progression, acute urinary retention, prostate-related surgeries, and costs in patients with benign prostatic hyperplasia taking early versus delayed combination $5 \alpha$-reductase inhibitor therapy and $\alpha$-blocker therapy: a retrospective analysis," Clinical Therapeutics, vol. 35, no. 5, pp. 624-633, 2013.

[10] J. D. McConnell, C. G. Roehrborn, O. M. Bautista et al., "The long-term effect of doxazosin, finasteride, and combination therapy on the clinical progression of benign prostatic hyperplasia," The New England Journal of Medicine, vol. 349, no. 25, pp. 2387-2398, 2003.

[11] C. G. Roehrborn, P. Siami, J. Barkin et al., "The effects of combination therapy with dutasteride and tamsulosin on clinical outcomes in men with symptomatic benign prostatic hyperplasia: 4-year results from the CombAT study," European Urology, vol. 57, pp. 123-131, 2010.
[12] R. Pickard, M. Emberton, and D. E. Neal, "The management of men with acute urinary retention," British Journal of Urology, vol. 81, no. 5, pp. 712-720, 1998.

[13] K. Murray, A. Massey, and R. C. L. Feneley, "Acute urinary retention-a urodynamic assessment," British Journal of Urology, vol. 56, no. 5, pp. 468-473, 1984.

[14] M. Oelke, A. Bachmann, A. Descazeaud et al., "EAU guidelines on the treatment and follow-up of non-neurogenic male lower urinary tract symptoms including benign prostatic obstruction," European Urology, vol. 64, no. 1, pp. 118-140, 2013.

[15] M. Maldonado-Ávila, H. A. Manzanilla-García, J. A. SierraRamírez et al., "A comparative study on the use of tamsulosin versus alfuzosin in spontaneous micturition recovery after transurethral catheter removal in patients with benign prostatic growth," International Urology and Nephrology, vol. 46, no. 4, pp. 687-690, 2014.

[16] K. Akiyama, H. Noto, O. Nishizawa et al., "Effect of KMD-3213, an $\alpha 1 \mathrm{~A}$-adrenoceptor antagonist, on the prostatic urethral pressure and blood pressure in male decerebrate dogs," International Journal of Urology, vol. 8, no. 4, pp. 177-183, 2001.

[17] H. Miyakita, E. Yokoyama, Y. Onodera et al., "Short-term effects of crossover treatment with silodosin and tamsulosin hydrochloride for lower urinary tract symptoms associated with benign prostatic hyperplasia," International Journal of Urology, vol. 17, no. 10, pp. 869-875, 2010.

[18] L. Annemans, I. Cleemput, M. Lamotte, A. McNeill, and T. Hargreave, "The economic impact of using alfuzosin $10 \mathrm{mg}$ once daily in the management of acute urinary retention in the UK: a 6-month analysis," BJU International, vol. 96, no. 4, pp. 566-571, 2005. 


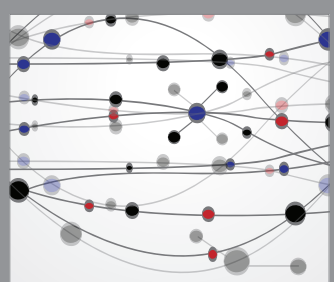

The Scientific World Journal
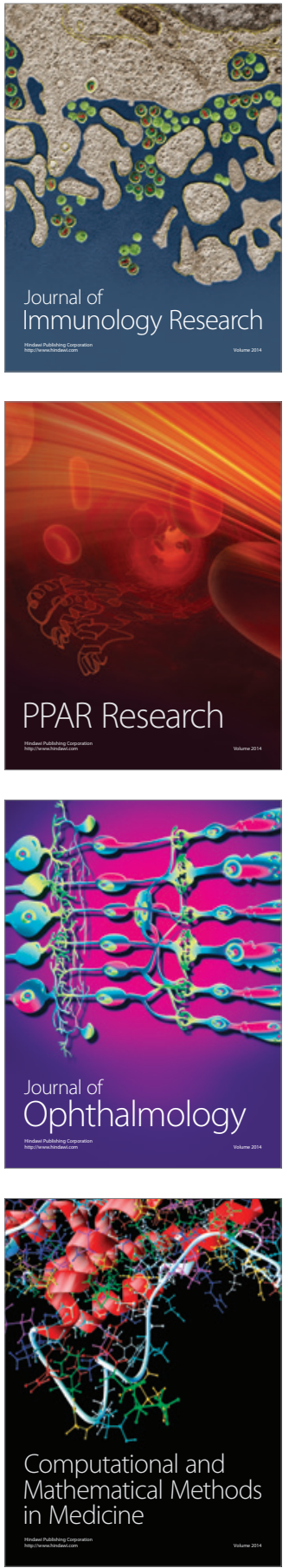

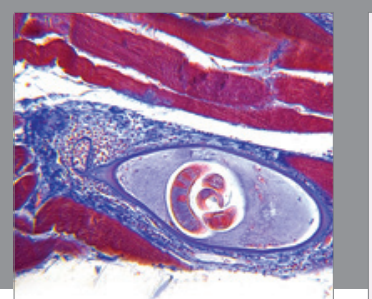

Gastroenterology Research and Practice

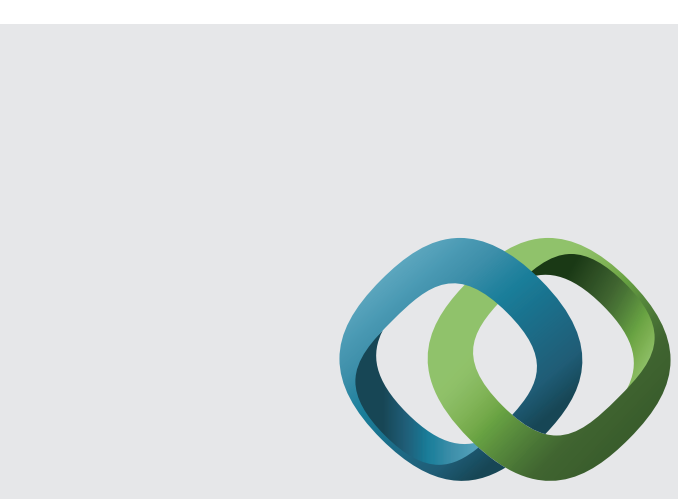

\section{Hindawi}

Submit your manuscripts at

http://www.hindawi.com
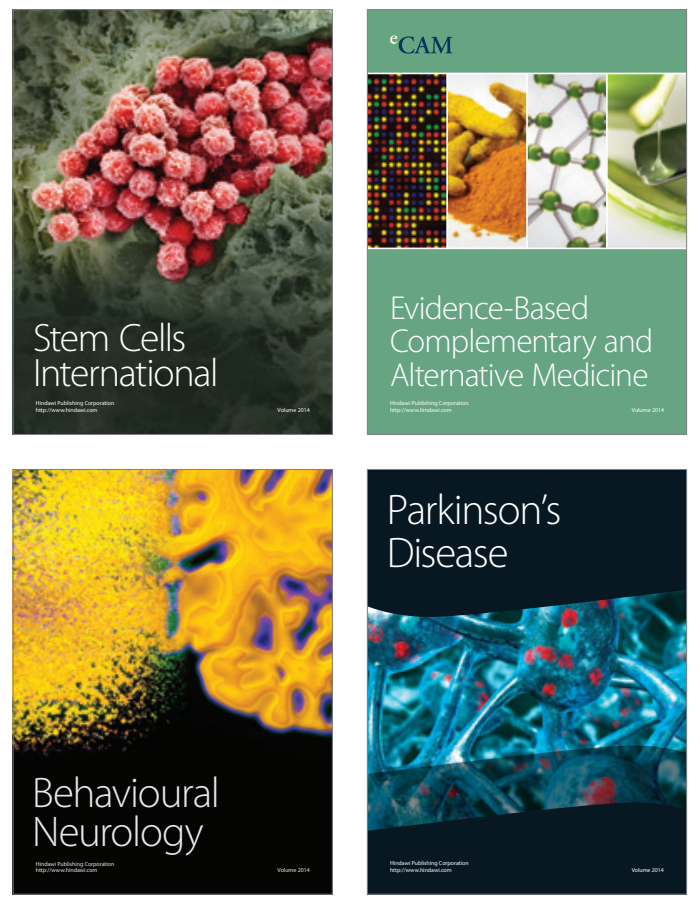
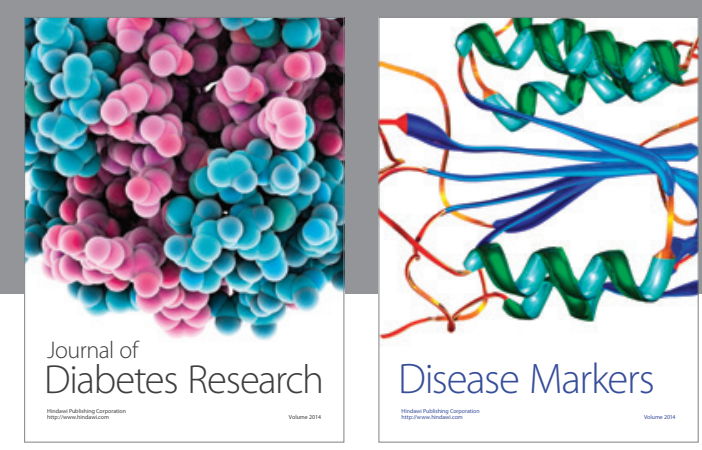

Disease Markers
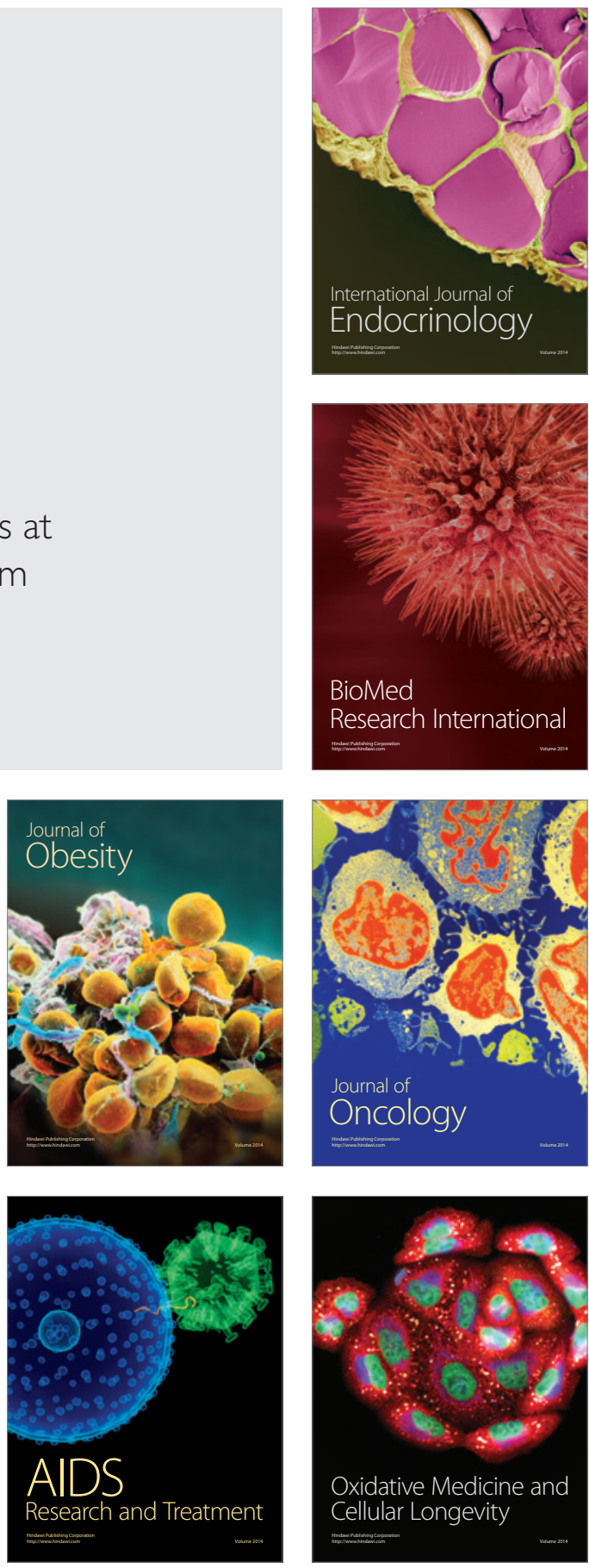